\title{
Global Shape from Shading for an Endoscope Image
}

\author{
S. Y. Yeung ${ }^{1}$, H. T. Tsui ${ }^{1}$, and A. Yim ${ }^{2}$ \\ 1 Department of Electronic Engineering \\ The Chinese University of Hong Kong, Shatin, Hong Kong \\ 2 Department of Surgery \\ Prince of Wales Hospital, ShaTin, Hong Kong
}

\begin{abstract}
Okatani and Deguchi [13] proposed a local Shape from Shading (SFS) method for endoscope images by assuming the point light, which is close to the projection center, to be at the projection center. We extended and modified their method and devised a global SFS algorithm for the reconstruction of the complex shape of an internal organ. Since the surface of an organ is not Lambertian in general, we obtained the bi-directional reflection distribution function (BRDF) curve by calibration using a robot arm to achieve accurate endoscope orientation and positioning. Inspired by the idea of Kimmel and Bruckstein [8], global SFS method is based on the identification of singular points on the distance map, which each has the surface normal pointing towards the light source. Equal distance contours are propagated from each singular point using a level set method to get a local distance map of the surface. This is repeated for all singular points. After that, a set of local distance maps are selected to be merged together to construct a global distance map using a new scheme. The shape of the object can then be obtained from the global distance map. Simulated and real experiments were performed to verify the algorithm. Experimental result of global SFS from a single real endoscope image of a human lung is quite good.
\end{abstract}

Keywords:Shape from Shading, Level set propagation, Endoscope image

\section{Introduction}

Shape from Shading (SFS) is a very useful technique for reconstructing 3D shape of an object from its single 2D grayscale image. When it was first introduced by Horn [4,5], some assumptions were made to simplify the analysis. For example, the light source is supposed to be a single point light located at a great distance from the surface. The surface is of Lambertian, which means that the incident light is reflected evenly to all directions in a hemisphere by a Lambertian surface. The intensity of the surface in the image depends on the angle between local surface normal and the light source direction. Besides, this method suffers from the problem of topological ambiguities, which is known to be the problem of global shape from shading. We must solve this problem in order to get a correct

C. Taylor, A. Colchester (Eds.): MICCAI'99, LNCS 1679, pp. 318-327, 1999.

(C) Springer-Verlag Berlin Heidelberg 1999 
global surface. These assumptions more or less limit the practical applications of Shape-from-Shading technique.

Many developments have been made to release these limitations. More accurate and complicated surface models are proposed to solve the problem of non-Lambertian surface. Torrance and Sparrow [18] proposed a theoretic reflectance model that takes specular reflection as well as diffuse reflection into consideration. Cook [3] extended the model by including ambient light and spectral dependencies. Bidirectional Reflection Distribution Function ( or BRDF for short ) is another model that can be used to describe conveniently the surface reflection by natural materials $[7,6,11,12]$.

It is very useful for morphological analysis of tumors on human inner organs if the global 3D shape can be reconstructed from endoscope image by SFS. However, the surface of human organ cannot be regarded as Lambertian. Okatani and Deguchi [13] used BRDF to describe the reflectance property to tackle this problem. They extended Kimmel and Bruckstein's SFS algorithm [10,9] and introduced a notation of equal distance contour to recover the 3D shape by propagating the contour using a Level Set method [16]. Their algorithm also tackled the problem of a near light source quite well. But the algorithm can not reconstruct the shape if it has several hills and valleys. It is very important and significant to have a global SFS algorithm for endoscope image because the surface of human inner organ is often complicated in shape.

Bricault et al. [1] proposed a model-based SFS algorithm for transbronchial biopsy. Their algorithm can reconstruct global 3D surface. But pre-operative CT scan data is required as model for 3D reconstruction. Global SFS problem has also been studied by J.Oliensis and P.Dupuis [14,15] and Kimmel [8]. Oliensis's algorithm is based on establishing the equivalence of shape from shading to a calculus of variations/optimal control problem. Kimmel's approach is based on classification of singular points according to topological properties of simple smooth surfaces. Both of the above algorithms assume a parallel light or a point light source distant from the surface. As far as we know, there is no method in the literature that can reconstruct global 3D shape from single grayscale image under a near light source. We extend Kimmel's algorithm and make it applicable to near light source.

The global SFS algorithm for endoscope image consists of three steps: First, starting from singular points, use a level set propagation algorithm to obtain distance maps from each surface point to the light source. This is the so-called local SFS problem for endoscope image. In this case, the singular points are those surface points that have local maximum intensity. Second, merge the distance maps together based on the classification of singular points. Third, project the distance map back to 3D coordinate to get the depth map of the object.

The rest of the paper is organized as follows. A brief review of local SFS algorithm for endoscope image by propagating equal distance contour proposed by Okatani etc. [13] is given in Section 2. In Section 3, the global SFS method for parallel light by Kimmel [8] is also reviewed, followed by the global SFS 
algorithm for endoscope image. The simulation and experiment results are shown in Section 4. Conclusion is given in Section 5.

\section{Local SFS Algorithm for Endoscope Image}

A pinhole camera model is used for endoscope. In addition, since the surface of human inner organ is not Lambertian, a more general reflection model, BRDF is used to describe the reflection property of the surface, taking both the incident and reflection direction into consideration. The light of the endoscope can be approximated by a point light source located at the camera projection center [13]. This configuration simplifies the problem of near point light source and BRDF a lot.

\subsection{Imaging System and Brightness Formulation}

Here, we follow the derivation of Okatani and Deguchi [13]. The grayscale $E$ in the image position $(x, y)$ is given by,

$$
E(x, y)=\delta \frac{F\left(\theta_{i}, \theta_{r}, \phi_{r}\right) \cos \theta_{i}}{r^{2}}
$$

where $F\left(\theta_{i}, \theta_{r}, \phi_{r}\right)$ is the bidirectional reflectance distribution function [7] of the surface. $\phi_{r}$ is the azimuth angle between the incident light and the reflection light. $r$ is the distance between the light source and the surface point, and $\delta$ is a constant depending on the intensity of light source and the camera parameters.

Since the light source and the view point is at the same position, the incident angle $\theta_{i}$ always equals to reflection angle $\theta_{r}$, and $\phi_{r}$ is 0 . Substitute the angles into Eq.1 yields,

$$
E(x, y)=\delta \frac{F\left(\theta_{i}, \theta_{i}, 0\right) \cos \theta_{i}}{r^{2}}=\delta \frac{G\left(\cos \theta_{i}\right)}{r^{2}}
$$

where $G\left(\cos \theta_{i}\right)=F\left(\theta_{i}, \theta_{i}, 0\right) \cos \theta_{i}$ is a monotonic increasing function with respect to $\cos \theta_{i}$. So given the image brightness $E(x, y)$ and the distance $r$, $\cos \theta_{i}$ can be uniquely determined by,

$$
\cos \theta_{i}=G^{-1}\left(r^{2} E(x, y) / \delta\right) .
$$

\subsection{Equal Distance Contour Propagation and Shape Reconstruction}

Equal distance contour may be thought of as a curve on the object surface, where all points on that curve have the same distance to the light source. the contour can also be regarded as the intersection of the object with a sphere whose center is the light source. Given an initial equal distance contour, we can propagate it to construct a new equal distance contour $r+\delta r$, with $\delta r$ small enough. Iteratively propagate the equal distance contour until each image point gets its distance to 
the projection center, the shape of the surface can then be represented by its distance map from the projection center of the endoscope. Starting from a small circle around a singular point as the initial equal distance contour $C\left(r_{0}\right)$, we define a function $\phi\left(x, y, r_{0}\right)$ on the image plane to be nagative inside the initial contour, and positive outside the contour. The equal distance contour at $r=t$ is given by

$$
C(t)=(x, y) \mid \phi(x, y, t)=0
$$

The contour can thus be propagated by updating the $\phi$ function with a velocity as

$$
\phi_{t}=-\frac{G^{-1}\left(t^{2} E(x, y) / \delta\right)}{f}
$$

where $f$ is the focal length of the endoscope.

Assume that the surface is smooth and simple and there is a point on the surface with the normal in the direction of the light source and has a minimum distance to the light. According to Eq.2, such a point can be selected by searching for the image point $\left(x_{0}, y_{0}\right)$ with maximum brightness $E\left(x_{0}, y_{0}\right)$. The boundary of a small neighborhood of that singular point serves as the intial distance contour. The intial distance is given by,

$$
r_{0}=\sqrt{\delta G(1) / E\left(x_{0}, y_{0}\right)}
$$

where the constant $\delta$ can be obtained by careful calibration.

\section{Global SFS Algorithm for Endoscope Image}

Distance map is an alternative form to represent the shape of an object. If we can obtain the distance map of a global surface from its endoscope image, we can get the shape of the object easily. So the global SFS problem is now how to obtain a global distance map of the surface. Local SFS should be used to get locally correct distance map propagating from each singular point. The local distance maps are then merged together to obtain a global solution.

\subsection{A Global Shape from Shading Algorithm for a Parallel Light}

A simple global algorithm for SFS with a parallel light is proposed by Kimmel etc. see also [8]. Assuming that the surface is simple and smooth and can be described by a Morse function [2]. All singular points on that surface can be locally classified to be three types: Minima, Maxima and Saddle. The singular points are those that have surface normal in the direction of light source, and can be determined by considering the grid point of highest intensity within a connected area of pixels.

By propagating the equal height contour from a singular point $m_{i}$ which is known to be a minima. we can obtain distance transform $D_{i}(x, y)$ of that singular point. The distance transform $D_{i}(x, y)$ is a relative depth map with respect to 
the singular point $m_{i}$. Given the absolute height $H\left(m_{i}\right)$ of each minima singular point on the depth map, the global surface $D_{r}(x, y)$ is given by [8],

$$
D_{r}(x, y)=\min \left(D_{i}(x, y)+H\left(m_{i}\right)\right): i=1 \text { to } N
$$

where $\mathrm{N}$ is the number of singular points which are minima. $D_{i}(x, y)+H\left(m_{i}\right)$ is the absolute height of the grid point $(x, y)$ on the depth map.

It is difficult to use Eq.7 directly to get the global depth map of a surface because the absolute height $H\left(m_{i}\right)$ of the minima singular point $m_{i}$ on the depth map is unknown. Besides, we cannot classify different types of singular points directly from the grayscale image, because all of the singular points are local brightest points. Kimmel proposed an iterative appoach to solve this problem. The inflection-type saddle point between two minima singular points should be identified. It serves as the merge point to merge two relative depth maps. The merging process goes on until a surface with no inflection-type saddle points is obtained. That surface is the global surface required. See [8] for detail.

\subsection{The Relationship between Depth Map and Distance Map}

Let us first examine the relationship between depth map of a surface and distance map of that surface to the camera projection center. Take a simple surface of a hemisphere for example, as shown in the Fig.1. The radius of that hemosphere is $R$, and the light source and camera are located at the center of the hemisphere. All points on the surface have the same distance $R$ to the light source. The corresponding distance map is shown in Fig.1(b). If on the depth map the surface normal at a point is in the direction of the light, its corresponding surface normal on the distance map is perpendicular to the $X-Y$ plane. It can be easily derived that propagating "equal distance contour" on the depth map is equivalent to the propagation of "equal height contour" on the distance map with a parallel light source coming from above and perpendicular to $X-Y$ plane. This equivalence helps to extend a global SFS algorithm for parallel light to a global SFS algorithm for a near light source.

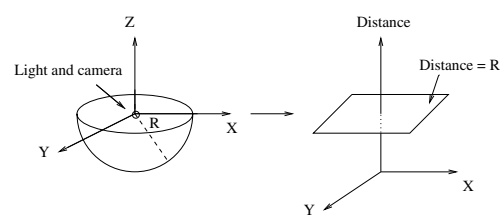

(a) Depth map $\quad$ (b) Distance map

Fig. 1. Relationship of depth map and distance map 


\subsection{A Global Shape from Shading Algorithm for Endoscope Image}

As mentioned before, the first step to reconstruct the global depth map of a surface from its endoscope image is to obtain its global distance map to the camera project center. We have discussed that propagating the equal distance contour on depth map is equivalent to propagating the "equal height contour" on the distance map. So the problem of obtaining global distance map for surface under endoscope is similar to the problem of obtaining global depth map for parallel light.

Assuming that the distance map is a simple and smooth surface. There are also three types of singular points: minima, maxima and saddle. (All singular points discussed below are on the distance map.) Starting from a minima singular point $m_{i}$, and using the Level set propagation, we can obtain a distance map $D_{i}(x, y)$. Since the absolute height of the point $m_{i}$ on the distance map can be estimated by Eq.6, the distance map $D_{i}(x, y)$ is an absolute distance map. The global distance map $D_{r}(x, y)$ in this case is given by

$$
D_{r}(x, y)=\min \left(D_{i}(x, y)\right), i=1 \text { to } N,
$$

The merging process is very simple. No iterative approach is needed. The resulting global distance map can be obtained from local distance maps in one step.

The key of this algorithm is to select all of the minima singular points on the distance map of the surface from the grayscale image. Let's have a look at the properties of three types of singular points on the distance map: Minima, Maxima and Saddle in the following.

Detecting Maxima and Saddle Points According to mountaineers' theorem [17], the number of extrema located within a closed equal-height contour of a smooth surface exceeds by one the number of saddle points within that contour. Start propagating from a small circle around a minima singular point, the first singular point met by the equal-height contour must be a saddle point. Assuming there is a minima singular point $m_{i}$ on the distance map, propagate equal-height contour from that point will yield a distance map $D_{i}(x, y)$. The first singular point met in the process of propagation must be a saddle point. Let us label the point as $S$. The height of that saddle point on the distance map can be correctly obtained from the distance map as $D_{i}\left(x_{s}, y_{s}\right)$, where $\left(x_{s}, y_{s}\right)$ is the coordinate of point $S$ in the image. As mentioned before, the surface normal at singular point will point in the direction of the light source. So $\cos \theta_{i}(S)$ equals 1 . The distance from that singular point to the light source can be estimated by [13],

$$
r_{0}\left(x_{s}, y_{s}\right)=\sqrt{\delta G(1) / E\left(x_{s}, y_{s}\right)}
$$

the value of $D_{i}\left(x_{s}, y_{s}\right)$ and $r_{0}\left(x_{s}, y_{s}\right)$ should match, i.e.

$$
\left|D_{i}\left(x_{s}, y_{s}\right)-r_{0}\left(x_{s}, y_{s}\right)\right|<\text { threshold }
$$


The value of $\left|D_{i}\left(x_{s}, y_{s}\right)-r_{0}\left(x_{s}, y_{s}\right)\right|$ may not be zero because there may be some small error in estimating $r_{0}\left(x_{s}, y_{s}\right)$ and obtaining $D_{i}\left(x_{s}, y_{s}\right)$.

When the equal height contour on the distance map propagates through the saddle point $S$, there are two different cases. The contour will meet a maxima point $(X)$ or a minima point $(N)$. If the next singular point met is a maxima, the contour will be split into two parts: the inner part of the contour will end at the maxima singular point, and get the height of that maxima point on the distance map correctly. The height of that maxima point obtained from the distance map $D_{i}(X)$ should match the distance estimated by Eq.6, just like the case of the saddle. If the contour after passing the saddle point meets another minima point, there will be a topological error, the height of the next minima obtained from the distance map is not correct. The difference between $D_{i}\left(x_{n}, y_{n}\right)$ and $r_{0}\left(x_{n}, y_{n}\right)$ will be large. This helps to identify minima singular points from maxima and saddle points.

The Proposed Global SFS Algorithm The procedure to get global distance map should be as follows:

step 1: Select singular points of distance map from the image by considering the local brightest point within a connected area of pixels in the image. These singular points, denoted as $m_{i}$, can fall into three types: minima, maxima and saddle.

step 2: Estimate the distance of each singular point $m_{i}$ to the light by Eq.6. Use a small circle around the singular point to be an initial contour. Propagate the equal distance contour to get a local distance map $D_{i}$ for each singular point.

step3: Use the obtained local distance maps to classify the singular points. Local distance maps propagated from maxima or saddle points must be deleted.

step 4: Merge the local distance maps starting from minima singular points together to get the global distance of the surface.

The procedure for the above step 2 is: For each singular point $m_{i}$, estimate its distance $r\left(m_{i}\right)$ to the light by Eq.6. Then search the distance map $D_{k}$ starting from other singular point. If there is a distance map $D_{k}$ such that $\mid D_{k}\left(m_{i}\right)-$ $r\left(m_{i}\right) \mid$ is smaller than a threshold, then the singular point $m_{i}$ is not a minima singular point. Then the corresponding distance map $D_{i}$ should be deleted. The threshold we used in our experiments is $\frac{1}{2}\left(r\left(m_{i}\right)-r\left(m_{k}\right)\right)$. The results are very good.

\section{Simulations and Experiments Results}

In the simulation, we use a software called Raytracing to simulate the endoscope lighting system and the camera. A complicated surface generated by matlab function $Z=P E A K S(X, Y)$ is used for testing. Its grayscale image taken by the simulated endoscope is shown in Fig.2(b). The global distance map was obtained first, as shown in Fig.2(c). The global depth map of the surface as shown in Fig.2(d) was then obtained by projecting the distance map back to 3D coordinate,. The result is very good. 


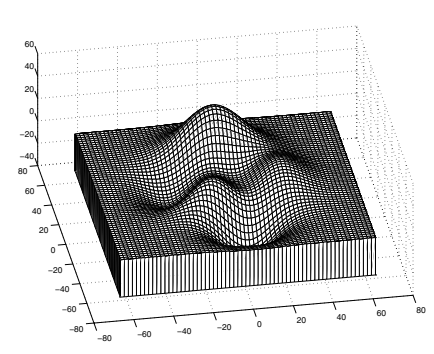

(a)Depth map of the original surface

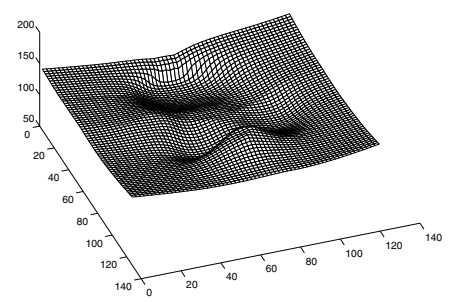

(c) Global distance map

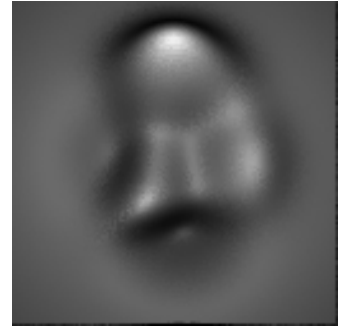

(b) Grayscale image of (a)

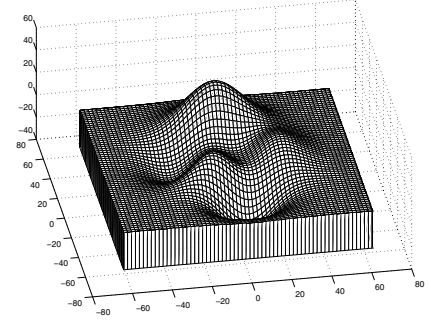

(d) Reconstructed global depth map

Fig. 2. Simulation result

Both Lambertian surface and nonLambertian surface are used in real experiments to verify the robustness of this algorithm. An image of a Lambertain model made of paper clay is taken by an real endoscope, as is shown in Fig.3(a). The reconstructed 3D surface of the model is shown in Fig.3(b). The BRDF for the Lambertian surface is degenerated to be $\cos \theta_{i}$. The result is satisfatory, except for little error due to interreflection between hills. Fig.4(a) shows the endoscope image of a patient's lung. Since the exact lung surface was not available for obtaining the BRDF, a similar surface was used to obtain the BRDF. A small piece of the surface was laid flat on the work table. The same endoscope was held by a robot arm to acquired images at viewing points forming a 90 degrees arc with the centre at the target object point. This allows us to compute a look up table for the function $G\left(\cos \theta_{i}\right)$. Shape of the lung was recovered using our global SFS method. Some liquid drops and blood drops are present on the image and had caused some unavoidable errors. In spite of this, the result is reasonably good.

\section{Conclusion}

In this paper, we have proposed a global SFS method for 3D reconstruction of an human internal organ from its endoscope image. Okatani and Deguchi [13] method is good only for local SFS. Unlike the method of Kimmel and Bruck- 


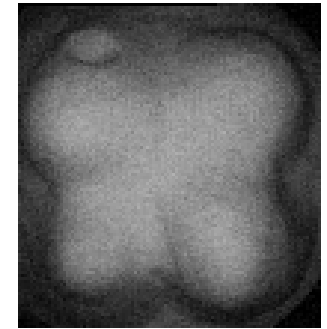

(a) Grayscale image

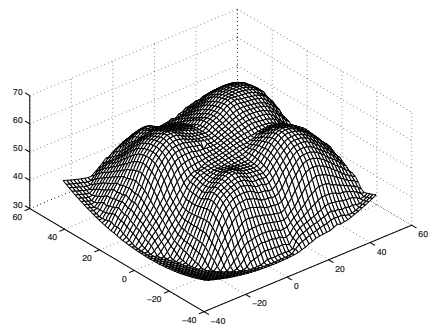

(b) Reconstructed surface

Fig. 3. Real experiment for Lambertian surface

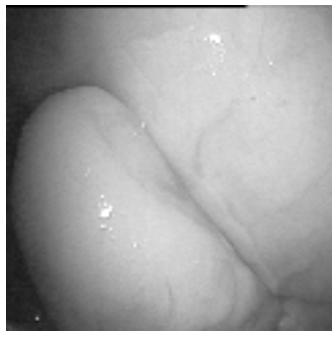

(a)Grayscale image

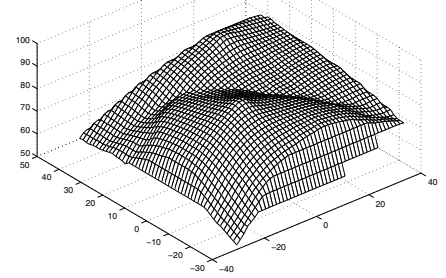

(b) Reconstructed surface

Fig. 4. Real experiment for non-Lambertian surface

stein [8] which used parallel light, our method used a near light model with the light assumed to be at the projection center. We obtained first a number of equal distance maps corresponding to the local minimum and merge them to form a global distance map. 3D shape is then derived from the global distance map. We did not assume the surface to be Lambertian. The BRDF of the surface is obtained by calibration with the help of a robot arm for accurate orientation and positioning of the endoscope. We have a different BRDF for different organ surface. Results from simulated experiments are very good. In the real images of organs, there are often bloods and liquid drops on the organ surface which have different surface reflectance properties. This heterogeneous surface is a major limitation of SFS in real applications. In spite of this, we managed to obtain quite good results. Better results may be expected if the blood and liquid can be removed from the image by image processing.

\section{Acknowledgements}

We thank Dr. T.W.Lee of the Prince of Wales Hospital for his help to get the real images for the experiment. This research was supported by the RGC research grants: CUHK 4162/97E and CUHK 4116/97E. 


\section{References}

1. I. Bricault, G. Ferretti, and P. Cinquin. "Multi-level Strategy for ComputerAssisted Transbronchial Biopsy". In Lecture Notes in Computer Science 1496, (MICCAI'98), pages 261-268, 1998. 319

2. J. W. Bruce and P. J. Giblin. "Curves and Singularities". 2nd ed. Cambridge Univ. Press, Cambridge, UK, 1992. 321

3. R. L. Cook and K. E. Torrance. "A Reflectance Model for Computer Graphics". Computer Graphics 15(3), pages 307-316, 1981. 319

4. B. K. P. Horn. "Shape from Shading: A method for Obtaining the Shape of a Smooth Opaque Object from One View". MIT Project MAC Internal Report TR79 and MIT AI Laboratoru Technical Report 232, November. 318

5. B. K. P. Horn. "Robot Vision". The MIT Press, 1986. 318

6. B. K. P. Horn and M. J. Brooks. "Shape from Shading". The MIT Press, 1989. 319

7. B. K. P. Horn and R. W. Sjoberg. "Calculating the reflection map". Applied Optics, 18, pages 1770-1779, 1979. 319, 320

8. R. Kimmel and A. Bruckstein. "Global Shape from Shading". Computer Vision and Image Understanding, Vol. 62, No. 3, pages 360-369, 1995. 318, 319, 321, 322, 326

9. R. Kimmel and A. Bruckstein. "Teacking Level Sets by Level Sets: A Method for Solving the Shape from Shading Problem". Computer Vision and Image Understanding, Vol. 62, No. 2, pages 47-58, 1995. 319

10. R. Kimmel, K. Siddiqi, B. Kimia, and A. Bruckstein. "Shape from Shading: Level Set Propagation and Viscosity Solutions". International Journal of Computer Vision, 16, pages 107-133, 1995. 319

11. S. K. Nayar, K. Ikeuchi, and T. Kanade. "Surface Reflection: Physical and Geometrical Perspectives". IEEE Transactions on Pattern Analysis and Machine Intelligence, pages 611-634, 1991. 319

12. F. E. Nicodemus, J. C. Richmond, J. J. Hsia, I. W. Girsberg, and T. Limperis. "Geometrical considerations and nomendature for reflectance". NBS Monograph 160 (National Bureau of standards, Washington, D.C.), October 1997. 319

13. T. Okatani and K. Deguchi. "Shape Reconstruction from an Endoscope Image by Shape from Shading Technique for a Point Light Source at the Projection Center". Computer Vision and Image Understanding, vol.66, No.2 May, pages 119-131, 1997. 318, 319, 320, 323, 325

14. J. Oliensis. "Shape from Shading as a Partially Well-Constrained Problem". CVGIP: Image Understanding, Vol. 54, pages 163-183, 1993. 319

15. J. Oliensis and P. Dupuis. "A Global Algorithm for Shape from Shading". In Proc. of the 4th ICCV, pages 692-701, 1993. 319

16. S. Osher and S. A. Sethian. "Fronts propagating with curvature-dependent speed: Algorithms based on Hamilton-Jacobi formulations". J. of Comput. Phys. 79, pages $12-49,1998.319$

17. E. Rouy and A. Tourin. "A viscosity solutions approach to shape-from shading". SIAM J. Number. Anal. 29(3), pages 867-884, June 1992. 323

18. K. E. Torrance and E. Sparrow. "Theory for off-Specular Reflection from Roughened Surface". J. Opt. Soc. Amer. 5\%, pages 1105-1114, 1967. 319 